\title{
Cycloaddition Reactions of Azatrienes with Sulfene
}

\author{
Parvesh Singh, Krishna Bisetty ${ }^{*}$ \\ Department of Chemistry, Durban University of Technology, Steve Biko Campus, Durban, South Africa \\ Email: parveshdurban@gmail.com, "bisettyk@dut.ac.za
}

Received March 10, 2012; revised April 16, 2012; accepted April 23, 2012

\begin{abstract}
Unprecedented cycloaddition reactions of azatrienes (1) with sulfene leading to the synthesis of functionalized thiazinedioxide derivatives (5) are described. The reactions were found be highly regioselective resulting in the formation of only [4+2] cycloadducts.
\end{abstract}

Keywords: Azadiene; Azatriene; Cycloaddition; Sulfene; Thiazine-Dioxide

\section{Introduction}

1-Azadienes are remarkably efficient precursors for the synthesis of nitrogen-containing heterocycles [1-10]. The presence of multiple reactive sites makes them excellent candidates for various synthetic manipulations. For example, $\alpha, \beta$-unsaturated imine can participate as a dienophile in a Michael type 1,4-addition (Rxn 1, Figure 1), or in a 1,2-addition (Rxn 2, Figure 1). Morever, depending upon the reaction partner, 1-azadiene can react as $2 \pi(\operatorname{Rxn} 3$, Figure 1) or $4 \pi$ (Rxn 4, Figure 1) component in the cycloaddition reactions. It is believed that the electron density in the 1-azadiene system is a significant factor that defines its reactivity. The presence of electrondonating groups $\left(-\mathrm{NR}_{2},-\mathrm{OR},-\mathrm{R},-\mathrm{OSi}\right)$ typically on nitrogen atom increases their reactivity towards electrophiles (Rxn 5, Figure 1) or electron deficient dienophiles in the hetero Diels-Alder (HDA) reactions [11-13]. On the other hand, the presence of electron-withdrawing groups (-COR, -COOR, $-\mathrm{SO}_{2}$ ) makes them prone to the attack of nucleophiles or electron rich dienophiles in the inverse electron-demand HDA reactions $[14,15]$. However, the $4 \pi$ participation of the 1-azadienes is reported to suffer from low conversion, competitive [2+2] addition, and low diene reactivity due to an unfavorable s-cis/s-trans equilibrium $[16,17]$. In addition the tautomerization of substituted 1azadienes to enamines precludes the $[4+2]$ cycloaddition due to the instability of endocyclic enamine products. Consequently, only a limited number of 1-aza-buta-1,3diene structural variations and modified or restricted reaction conditions have been introduced that have permitted the productive $4 \pi$ participation of $\alpha, \beta$-unsaturated imines in [4+2] cycloaddition reactions.

Literature study reveals that the cycloaddition reactions of highly reactive heterodienophile sulfene have not

\footnotetext{
${ }^{*}$ Corresponding author.
}

extensively been explored with the $\mathrm{C}=\mathrm{N}$ double bond [18]

Recently, we reported a single pot synthesis of stable cross-conjugated azatrienes 1 (Scheme 1) along with the tandem [2 +2$]$ cycloaddition and highly facile [3,3]-sigmatropic rearrangements in their reactions with conjugated ketenes, leading to facile synthesis of functionalized azocinone derivatives [19]. In connection with our ongoing interest in this research area, we widened our study to the cycloaddition reactions of azatrienes with sulfene dienophile. To the best of our knowledge, this is a first report where the [4+2] cycloadditions of 1-azadienes are carried out with the sulfene.

\section{Results and Discussion}

The cycloadditions were realized by the dropwise addition of a solution of methanesulfonyl chloride (2eq.) to a cooled dichloromethane solution of azatrienes $\mathbf{1}[19,20]$ and triethylamine (3eq.). The reactions were found to be highly regioselective leading to the exclusive formation of cycloadducts 5 without any traces of corresponding [2 +2 ] adducts 6 (Scheme 1).

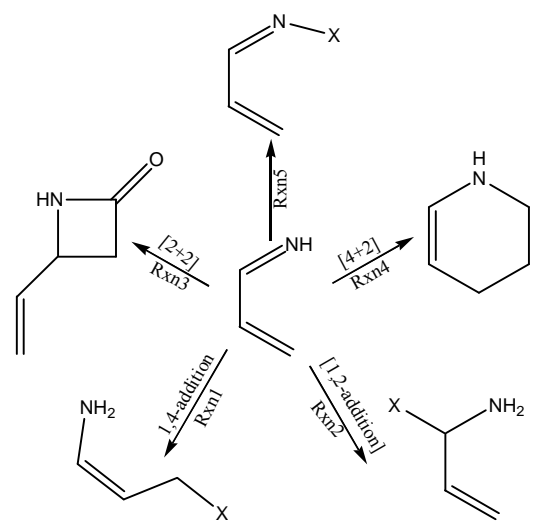

Figure 1. Different reaction types of 1-azadiene system. 


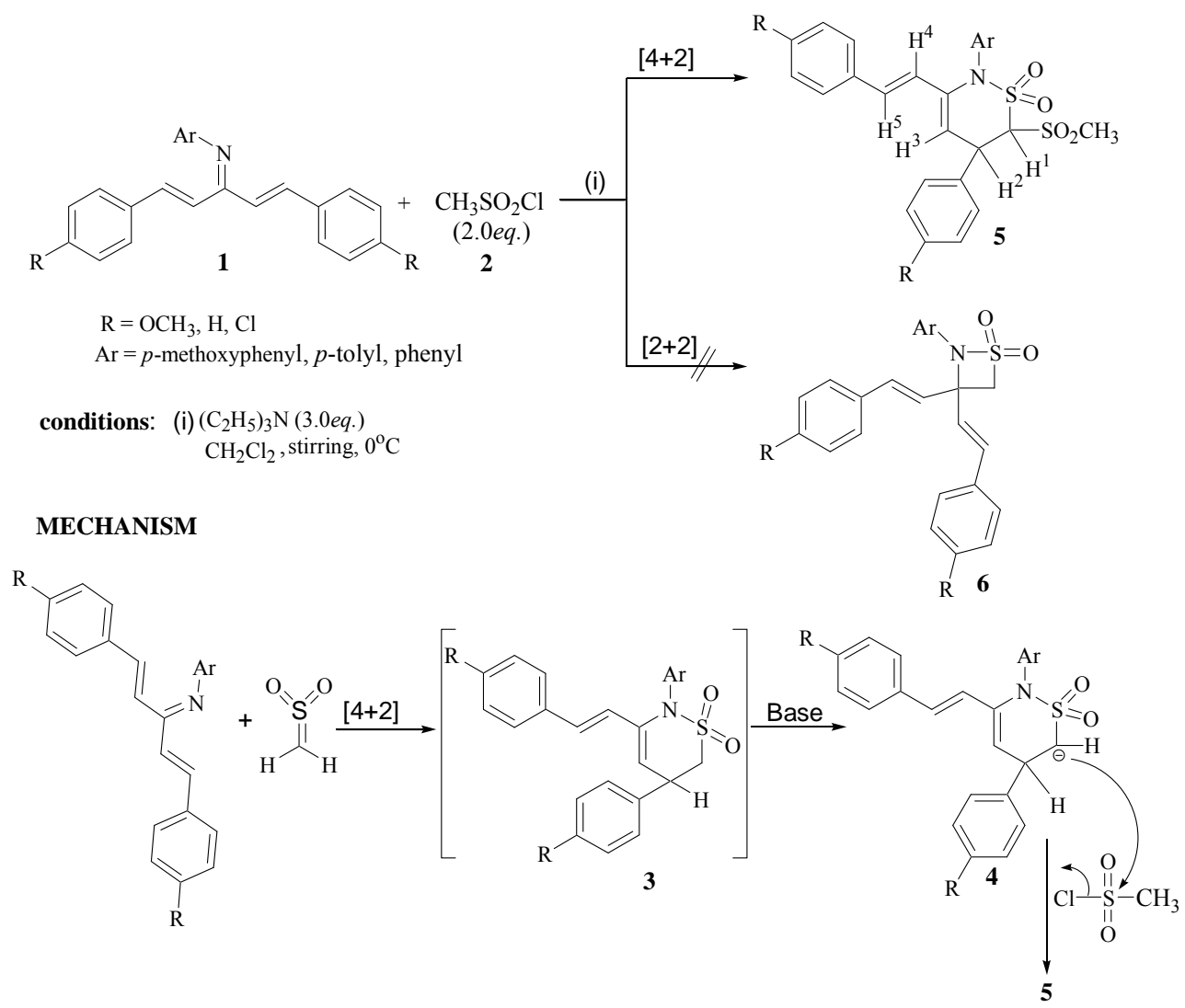

Scheme 1. Synthetic route and mechanism for the preparation of thiazine-dioxide derivatives from cycloaddition reactions of azatrienes (1) with sulfene.

The solid compounds, isolated after column chromatography, were characterized as thiazine-dioxide derivatives 5 on the basis of their available spectral data and analytical evidence. The plausible mechanistic pathway followed in these reactions involved the initial formation of [ $4+2]$ cycloadduct 3 as an intermediate that upon loss of proton under basic conditions get transformed into another intermediate 4 (Scheme 1). The intermediate 4 subsequently underwent sulfonylation under reaction conditions resulting in thiazine derivatives $\mathbf{5}$ in reasonable yields (Table 1). The treatment of azatrienes 5 with equimolar ratio of methanesulfonyl chloride and triethylamine led to the incompletion of reaction with the partial generation of compound 5 (TLC based), clearly ruling out any possibility of compound $\mathbf{6}$ formed in the reaction. Moreover, the reaction did not proceed between $\mathbf{5}$ and methanesulfonyl chloride (TLC based) in the absence of triethylamine.

The detailed spectral features of the compounds 5 are given in the experimental section; however, the significant features are mentioned here. The compound $\mathbf{5 a}$, for example, analyzed for $\mathrm{C}_{28} \mathrm{H}_{29} \mathrm{NO}_{7} \mathrm{~S}_{2}$, exhibited a molecular ion peak at $m / z 555\left(\mathrm{M}^{+}\right)$in its mass spectrum. The ${ }^{\mathrm{I}} \mathrm{H}$ spectrum showed a characteristic doublet at $\delta 4.51(J=$ $10.7 \mathrm{~Hz}$ ) for one methine proton $\left(\mathrm{H}^{1}\right)$, one doublet of dou- blet (dd) at $\delta 4.76(J=10.7 \mathrm{~Hz} \& 3.3 \mathrm{~Hz})$ for another methine proton $\left(\mathrm{H}^{2}\right)$, a doublet at $\delta 5.75(J=3.3 \mathrm{~Hz})$ for olefinic proton $\left(\mathrm{H}^{3}\right)$ and two doublets at $\delta 6.21$ and 6.52 $(J=16.0 \mathrm{~Hz})$ corresponding to two trans olefinic protons $\left(\mathrm{H}^{4} \& \mathrm{H}^{5}\right)$ respectively. The characteristic four singlets at $\delta 2.97,3.76,3.82$ and 3.83 confirmed the presence of single $-\mathrm{SO}_{2} \mathrm{CH}_{3}$ and three methoxy groups in the compound.

\section{Conclusion}

The highly regioselective cycloadditions reactions of azatrienes (1) with sulfene dienophile is described, and a series of new functionalized thiazine-dioxide scaffolds has been prepared. This is a first report, to the best of our knowledge, in which the $[4+2]$ cycloadditions of 1-azabutadienes are explored with the sulfene dienophile.

\section{Experimental Section}

\subsection{General}

The cross-conjugated azatrienes $\mathbf{1}$ were prepared according to the reported procedure $[19,20]$. Thionyl chloride and methanesulfonyl chloride used were commercially available. Dichloromethane dried over $d i$-phosphorus pentoxide and stored over molecular sieves (4 $\AA$ ). Mass 
Table 1. Reactions of azatrienes (1) with the sulfene.

\begin{tabular}{cccccc}
\hline S.No. & Compound & $\mathbf{R}$ & Ar & Yield (\%) & M.P. $\left({ }^{\circ} \mathbf{C}\right)$ \\
\hline 1 & $5 \mathrm{a}$ & $-\mathrm{OCH}_{3}$ & $p$-methoxyphenyl & 79 & $113-114$ \\
2 & $5 \mathrm{~b}$ & $-\mathrm{OCH}_{3}$ & $p$-tolyl & 76 & $134-135$ \\
3 & $5 \mathrm{c}$ & $-\mathrm{OCH}_{3}$ & phenyl & 69 & $121-122$ \\
4 & $5 \mathrm{~d}$ & $\mathrm{H}$ & $p$-methoxyphenyl & 77 & $118-119$ \\
5 & $5 \mathrm{e}$ & $\mathrm{H}$ & -tolyl & 68 & $125-126$ \\
6 & $5 \mathrm{f}$ & $\mathrm{H}$ & phenyl & 71 & $130-131$ \\
7 & $5 \mathrm{~g}$ & $\mathrm{Cl}$ & $\mathrm{Cl}$-methoxyphenyl & 75 & $136-137$ \\
8 & $5 \mathrm{~h}$ & $\mathrm{Cl}$ & phenyl & 72 & $132-133$ \\
9 & $5 \mathrm{i}$ & & & 78 & $116-117$ \\
\hline
\end{tabular}

spectra were recorded on Shimadzu GCMS-QP-2000 mass spectrometer. IR spectra were recorded on a Shimadzu D-8001 spectrophotometer. ${ }^{1} \mathrm{H}$ NMR spectra were recorded in deuterochloroform $\left(\mathrm{CDCl}_{3}\right)$ with Bruker $\mathrm{AC}$ E $300(300 \mathrm{MHz})$ spectrometer using tetramethylsilane (TMS) as an internal standard. Chemical shift values are expressed as ppm downfield from TMS and $J$ values are in Hz. Splitting patterns are indicated as s: singlet, d: doublet and dd: doublet of doublet. ${ }^{13} \mathrm{C}-\mathrm{NMR}$ spectra were also recorded on AC-E $300(75.0 \mathrm{MHz})$ spectrometer in a deuterochloroform $\left(\mathrm{CDCl}_{3}\right)$ using tetramethylsilane (TMS) as an internal standard. Melting points were determined by open capillary method using Veego Precision Digital Melting Point apparatus (MP-D) and are uncorrected. Elemental analyses were performed on Heraus CHN-O-Rapid Elemental Analyzer.

\subsection{General Procedure for the Cycloaddition Reactions of Azatrienes (1) with Sulfene}

To a well-stirred solution of azatrienes $1(10 \mathrm{mmol})$ and triethylamine $(30 \mathrm{mmol})$ in dry methylene chloride $(30$ $\mathrm{mL}$ ) was added drop wise a solution of methanesulfonyl chloride $(20 \mathrm{mmol})$ in dry methylene chloride $(30 \mathrm{~mL})$ over a period of 0.5 hour at $0^{\circ} \mathrm{C}$. After completion of the reaction (TLC), the reaction mixture was first washed with the saturated sodium bicarbonate solution $(2 \times 25$ $\mathrm{mL})$ and water $(2 \times 50 \mathrm{~mL})$ and the organic layer dried over anhydrous sodium sulfate. Removal of solvent under reduced pressure yielded the crude product, which was purified by silica gel column chromatography using a mixture of ethyl acetate and hexane $(2: 10, \mathrm{v} / \mathrm{v})$.

6-Methanesulfonyl-2,5-bis-(4-methoxy-phenyl)-3-[2 -(4-methoxy-phenyl)-vinyl]-5,6-dihydro-2H-[1,2]thiazi ne 1,1-dioxide (5a). Light yellow solid, yield 79\%; mp $113^{\circ} \mathrm{C}-114^{\circ} \mathrm{C}$; IR (KBr) 1604, 1510, $1463 \mathrm{~cm}^{-1} ;{ }^{1} \mathrm{H}$ NMR (300 MHz, $\mathrm{CDCl}_{3}$ ): $\delta 2.97\left(\mathrm{~s}, 3 \mathrm{H} .-\mathrm{SO}_{2} \mathrm{CH}_{3}\right), 3.76$ $\left(\mathrm{s}, 3 \mathrm{H},-\mathrm{OCH}_{3}\right), 3.82\left(\mathrm{~s}, 3 \mathrm{H},-\mathrm{OCH}_{3}\right), 3.83(\mathrm{~s}, 3 \mathrm{H}$, $\left.-\mathrm{OCH}_{3}\right), 4.51\left(\mathrm{~d}, J=10.7 \mathrm{~Hz}, 1 \mathrm{H}, \mathrm{H}^{1}\right), 4.76(\mathrm{dd}, J=10.7$ \& $\left.3.3 \mathrm{~Hz}, 1 \mathrm{H}, \mathrm{H}^{2}\right), 5.75\left(\mathrm{~d}, J=3.3 \mathrm{~Hz}, 1 \mathrm{H}, \mathrm{H}^{3}\right), 6.21(\mathrm{~d}$,
$\left.J=16.0 \mathrm{~Hz}, 1 \mathrm{H}, \mathrm{H}^{4}\right), 6.52\left(\mathrm{~d}, J=16.0 \mathrm{~Hz}, 1 \mathrm{H}, \mathrm{H}^{5}\right), 6.78$ (d, $J=8.6 \mathrm{~Hz}, 2 \mathrm{H}, \mathrm{ArH}), 6.97$ (d, $J=8.6 \mathrm{~Hz}, 2 \mathrm{H}, \mathrm{ArH})$, $7.15(\mathrm{~d}, J=8.6 \mathrm{~Hz}, 4 \mathrm{H}, \mathrm{ArH}), 7.36(\mathrm{~d}, J=8.7 \mathrm{~Hz}, 2 \mathrm{H}$, $\mathrm{ArH}), 7.44$ (d, $J=8.7 \mathrm{~Hz}, 2 \mathrm{H}, \mathrm{ArH}) \mathrm{ppm} ;{ }^{13} \mathrm{C} \mathrm{NMR}$ $\left(\mathrm{CDCl}_{3}\right): \delta 43.1(-\mathrm{CH}), 44.1\left(-\mathrm{SO}_{2} \mathrm{CH}_{3}\right), 55.2\left(-\mathrm{OCH}_{3}\right)$, $55.3\left(-\mathrm{OCH}_{3}\right), 55.4\left(-\mathrm{OCH}_{3}\right), 75.9(-\mathrm{CH}), 96.1,114.0$, $114.5,119.7,120.8,127.8,127.9,128.5,129.6,130.1$, $131.1,132.6,136.3,159.2,159.4,159.8$ ppm; MS (EI) $m / z$ : $555\left(\mathrm{M}^{+}\right)$. Anal. Calcd. for $\mathrm{C}_{28} \mathrm{H}_{29} \mathrm{NO}_{7} \mathrm{~S}_{2}$ : C, 60.52; H, 5.26; N, 2.52\%. Found: C, 60.71; H, 5.19; N, 2.47\%.

6-Methanesulfonyl-5-(4-methoxy-phenyl)-3-[2-(4methoxy-phenyl)-vinyl]-2-p-tolyl-5,6-dihydro-2H-[1,2] thiazine 1,1-dioxide (5b). Colorless solid, yield 76\%; $\mathrm{mp} 134^{\circ} \mathrm{C}-135^{\circ} \mathrm{C}$; IR (KBr) $1603,1510,1463 \mathrm{~cm}^{-1} ;{ }^{1} \mathrm{H}$ NMR $\left(300 \mathrm{MHz}, \mathrm{CDCl}_{3}\right): \delta 2.05\left(\mathrm{~s}, 3 \mathrm{H},-\mathrm{CH}_{3}\right), 3.00(\mathrm{~s}$, $3 \mathrm{H}$. $\left.-\mathrm{SO}_{2} \mathrm{CH}_{3}\right), 3.76\left(\mathrm{~s}, 3 \mathrm{H},-\mathrm{OCH}_{3}\right), 3.83\left(\mathrm{~s}, 3 \mathrm{H},-\mathrm{OCH}_{3}\right)$, $4.49\left(\mathrm{~d}, J=10.8 \mathrm{~Hz}, 1 \mathrm{H}, \mathrm{H}^{1}\right), 4.75(\mathrm{dd}, J=10.8 \& 3.4$ $\left.\mathrm{Hz}, 1 \mathrm{H}, \mathrm{H}^{2}\right), 5.77\left(\mathrm{~d}, J=3.4 \mathrm{~Hz}, 1 \mathrm{H}, \mathrm{H}^{3}\right), 6.22(\mathrm{~d}, J=$ $\left.16.0 \mathrm{~Hz}, 1 \mathrm{H}, \mathrm{H}^{4}\right), 6.50\left(\mathrm{~d}, J=16.0 \mathrm{~Hz}, 1 \mathrm{H}, \mathrm{H}^{5}\right), 6.77(\mathrm{~d}$, $J=8.6 \mathrm{~Hz}, 2 \mathrm{H}, \mathrm{ArH}), 6.96(\mathrm{~d}, J=8.6 \mathrm{~Hz}, 2 \mathrm{H}, \mathrm{ArH})$, 7.14 (d, $J=8.6 \mathrm{~Hz}, 2 \mathrm{H}, \mathrm{ArH}), 7.23-7.43$ (m, 6H, ArH) ppm; ${ }^{13} \mathrm{C} \mathrm{NMR}\left(\mathrm{CDCl}_{3}\right): \delta 21.0\left(-\mathrm{CH}_{3}\right), 43.2(-\mathrm{CH}), 44.2$ $\left(-\mathrm{SO}_{2} \mathrm{CH}_{3}\right), 55.3\left(-\mathrm{OCH}_{3}\right), 55.4\left(-\mathrm{OCH}_{3}\right), 76.1(-\mathrm{CH})$, $96.1,114.0,114.6,120.2,120.8,126.4,127.9,128.3$, $128.5,129.6,132.7,135.8,136.3,138.4,159.5,159.8$ ppm; MS (EI) $m / z: 539\left(\mathrm{M}^{+}\right)$. Anal. Calcd. for $\mathrm{C}_{28} \mathrm{H}_{29} \mathrm{NO}_{6} \mathrm{~S}_{2}$ : C, 62.32; H, 5.42; N, 2.60\%. Found: C, $62.25 ; \mathrm{H}, 5.51 ; \mathrm{N}, 2.53 \%$.

6-Methanesulfonyl-5-(4-methoxy-phenyl)-3-[2-(4methoxy-phenyl)-vinyl]-2-phenyl-5,6-dihydro-2H-[1,2] thiazine 1,1-dioxide (5c). Pale yellow solid, yield 69\%; mp $121^{\circ} \mathrm{C}-122^{\circ} \mathrm{C}$; IR (KBr) 1604, $1510,1462 \mathrm{~cm}^{-1} ;{ }^{1} \mathrm{H}$ NMR $\left(300 \mathrm{MHz}, \mathrm{CDCl}_{3}\right): \delta 2.99\left(\mathrm{~s}, 3 \mathrm{H} .-\mathrm{SO}_{2} \mathrm{CH}_{3}\right), 3.79$ $\left(\mathrm{s}, 3 \mathrm{H},-\mathrm{OCH}_{3}\right), 3.82\left(\mathrm{~s}, 3 \mathrm{H},-\mathrm{OCH}_{3}\right), 4.47(\mathrm{~d}, J=10.8$ $\left.\mathrm{Hz}, 1 \mathrm{H}, \mathrm{H}^{1}\right), 4.73\left(\mathrm{dd}, J=10.8 \& 3.3 \mathrm{~Hz}, 1 \mathrm{H}, \mathrm{H}^{2}\right), 5.76$ $\left(\mathrm{d}, J=3.3 \mathrm{~Hz}, 1 \mathrm{H}, \mathrm{H}^{3}\right), 6.21\left(\mathrm{~d}, J=16.0 \mathrm{~Hz}, 1 \mathrm{H}, \mathrm{H}^{4}\right)$, $6.51\left(\mathrm{~d}, J=16.0 \mathrm{~Hz}, 1 \mathrm{H}, \mathrm{H}^{5}\right), 6.79(\mathrm{~d}, J=8.6 \mathrm{~Hz}, 2 \mathrm{H}$, ArH), 6.94 (d, $J=8.6$ Hz, 2H, ArH), $7.11-7.46$ (m, 9H, $\mathrm{ArH}) \mathrm{ppm} ;{ }^{13} \mathrm{C} \mathrm{NMR}\left(\mathrm{CDCl}_{3}\right): \delta 42.9(-\mathrm{CH}), 44.1$ $\left(-\mathrm{SO}_{2} \mathrm{CH}_{3}\right), 55.1\left(-\mathrm{OCH}_{3}\right), 55.2\left(-\mathrm{OCH}_{3}\right), 76.0(-\mathrm{CH}), 96.1$, 
$114.0,114.3,120.1,121.2,125.8,127.4,128.2,129.3$, $130.8,132.1,134.9,135.8,138.6,159.2,159.6$ ppm; MS (EI) $m / z: 525\left(\mathrm{M}^{+}\right)$. Anal. Calcd. for $\mathrm{C}_{27} \mathrm{H}_{27} \mathrm{NO}_{6} \mathrm{~S}_{2}$ : C, 61.69; H, 5.18; N, 2.66\%. Found: C, 61.78; H, 5.11; N, $2.56 \%$.

6-Methanesulfonyl-2-(4-methoxy-phenyl)-5-phenyl3-styryl-5,6-dihydro-2H-[1,2]thiazine 1, 1-dioxide (5d). Yellow solid, yield $77 \%$; mp $118^{\circ} \mathrm{C}-119^{\circ} \mathrm{C}$; IR (KBr) 1607, 1517, $1460 \mathrm{~cm}^{-1} ;{ }^{1} \mathrm{H}$ NMR (300 MHz, $\left.\mathrm{CDCl}_{3}\right): \delta$ 3.01 (s, 3H. $\left.-\mathrm{SO}_{2} \mathrm{CH}_{3}\right), 3.82\left(\mathrm{~s}, 3 \mathrm{H},-\mathrm{OCH}_{3}\right), 4.57$ (d, $J=$ $\left.10.5 \mathrm{~Hz}, 1 \mathrm{H}, \mathrm{H}^{1}\right), 4.77\left(\mathrm{dd}, J=10.5 \& 2.9 \mathrm{~Hz}, 1 \mathrm{H}, \mathrm{H}^{2}\right)$, $5.82\left(\mathrm{~d}, J=2.9 \mathrm{~Hz}, 1 \mathrm{H}, \mathrm{H}^{3}\right), 6.34(\mathrm{~d}, J=16.0 \mathrm{~Hz}, 1 \mathrm{H}$, $\left.\mathrm{H}^{4}\right), 6.58\left(\mathrm{~d}, J=16.0 \mathrm{~Hz}, 1 \mathrm{H}, \mathrm{H}^{5}\right), 6.85(\mathrm{~d}, J=8.5 \mathrm{~Hz}$, 2H, ArH), 6.97 (d, J=8.5 Hz, 2H, ArH), 7.22 - 7.44 (m, $10 \mathrm{H}, \mathrm{ArH}) \mathrm{ppm} ;{ }^{13} \mathrm{C} \mathrm{NMR}\left(\mathrm{CDCl}_{3}\right): \delta 42.7(-\mathrm{CH}), 43.8$ $\left(-\mathrm{SO}_{2} \mathrm{CH}_{3}\right), 55.4\left(-\mathrm{OCH}_{3}\right), 76.1(-\mathrm{CH}), 96.0,113.8,114.2$, $119.9,122.8,125.4,126.3,128.1,128.4,130.2,131.7$, 132.8, 133.6, 135.9, 155.1, 159.8 ppm; MS (EI) $\mathrm{m} / \mathrm{z}: 495$ $\left(\mathrm{M}^{+}\right)$. Anal. Calcd. for $\mathrm{C}_{26} \mathrm{H}_{25} \mathrm{NO}_{5} \mathrm{~S}_{2}$ : C, 63.01; H, 5.08; N, 2.83\%. Found: C, 62.94; H, 5.17; N, 2.76\%.

6-Methanesulfonyl-5-phenyl-3-styryl-2-p-tolyl-5,6-d ihydro-2H-[1,2]thiazine1,1-dioxide (5e). Colorless solid, yield $68 \%$; mp $125^{\circ} \mathrm{C}-126^{\circ} \mathrm{C}$; IR (KBr) 1605,1511 , $1460 \mathrm{~cm}^{-1} ;{ }^{1} \mathrm{H}$ NMR $\left(300 \mathrm{MHz}, \mathrm{CDCl}_{3}\right): \delta 2.12(\mathrm{~s}, 3 \mathrm{H}$, $\left.-\mathrm{CH}_{3}\right), 3.00$ (s, 3H. $\left.-\mathrm{SO}_{2} \mathrm{CH}_{3}\right), 4.55$ (d, $J=10.6 \mathrm{~Hz}, 1 \mathrm{H}$, $\left.\mathrm{H}^{1}\right), 4.74\left(\mathrm{dd}, J=10.6 \& 3.1 \mathrm{~Hz}, 1 \mathrm{H}, \mathrm{H}^{2}\right), 5.82(\mathrm{~d}, J=$ $\left.3.1 \mathrm{~Hz}, 1 \mathrm{H}, \mathrm{H}^{3}\right), 6.33\left(\mathrm{~d}, J=16.0 \mathrm{~Hz}, 1 \mathrm{H}, \mathrm{H}^{4}\right), 6.54(\mathrm{~d}, J$ $\left.=16.0 \mathrm{~Hz}, 1 \mathrm{H}, \mathrm{H}^{5}\right), 6.92(\mathrm{~d}, J=8.6 \mathrm{~Hz}, 2 \mathrm{H}, \mathrm{ArH}), 7.10$ (d, $J=8.6 \mathrm{~Hz}, 2 \mathrm{H}, \mathrm{ArH}), 7.15-7.48$ (m, 10H, ArH) ppm; ${ }^{13} \mathrm{C}$ NMR $\left(\mathrm{CDCl}_{3}\right): \delta 20.9\left(-\mathrm{CH}_{3}\right), 42.4(-\mathrm{CH}), 44.1$ $\left(-\mathrm{SO}_{2} \mathrm{CH}_{3}\right), 76.3(-\mathrm{CH}), 96.1,114.1,114.6,120.4,123.1$, $124.9,126.1,128.6,129.5,130.2,130.8,131.6,133.2$, 133.9, 142.7, 159.5 ppm; MS (EI) $\mathrm{m} / z: 479\left(\mathrm{M}^{+}\right)$. Anal. Calcd. for $\mathrm{C}_{26} \mathrm{H}_{25} \mathrm{NO}_{4} \mathrm{~S}_{2}$ : C, 65.11; H, 5.25; N, 2.92\%. Found: C, 65.23; H, 5.17; N, 2.86\%.

6-Methanesulfonyl-2,5-diphenyl-3-styryl-5,6-dihydr o-2H-[1,2]thiazine 1,1-dioxide (5f). Colorless solid, yield $71 \%$; mp $130^{\circ} \mathrm{C}-131^{\circ} \mathrm{C}$; IR (KBr) $1603,1510,1460$ $\mathrm{cm}^{-1} ;{ }^{1} \mathrm{H}$ NMR $\left(300 \mathrm{MHz}, \mathrm{CDCl}_{3}\right): \delta 3.01(\mathrm{~s}, 3 \mathrm{H}$. $\left.-\mathrm{SO}_{2} \mathrm{CH}_{3}\right), 4.54\left(\mathrm{~d}, J=10.5 \mathrm{~Hz}, 1 \mathrm{H}, \mathrm{H}^{1}\right), 4.71(\mathrm{dd}, J=$ $\left.10.5 \& 2.8 \mathrm{~Hz}, 1 \mathrm{H}, \mathrm{H}^{2}\right), 5.82\left(\mathrm{~d}, J=2.8 \mathrm{~Hz}, 1 \mathrm{H}, \mathrm{H}^{3}\right)$, $6.36\left(\mathrm{~d}, J=16.0 \mathrm{~Hz}, 1 \mathrm{H}, \mathrm{H}^{4}\right), 6.53(\mathrm{~d}, J=16.0 \mathrm{~Hz}, 1 \mathrm{H}$, $\left.\mathrm{H}^{5}\right), 6.79-7.53(\mathrm{~m}, 15 \mathrm{H}, \mathrm{ArH}) \mathrm{ppm} ;{ }^{13} \mathrm{C} \mathrm{NMR}\left(\mathrm{CDCl}_{3}\right)$ : $\delta 42.9(-\mathrm{CH}), 44.4\left(-\mathrm{SO}_{2} \mathrm{CH}_{3}\right), 76.6(-\mathrm{CH}), 96.1,114.0$, $114.5,120.6,121.5,122.5,124.7,126.7,129.2,130.5$, 132.4, 133.4, 133.4, 135.7, 144.7, 159.6 ppm; MS (EI) $m / z: 465\left(\mathrm{M}^{+}\right)$. Anal. Calcd. for $\mathrm{C}_{25} \mathrm{H}_{23} \mathrm{NO}_{4} \mathrm{~S}_{2}$ : C, 64.49; H, 4.98; N, 3.01\%. Found: C, 64.59; H, 5.10; N, 2.96\%.

5-(4-Chloro-phenyl)-3-[2-(4-chloro-phenyl)-vinyl]-6 -methanesulfonyl-2-(4-methoxy-phenyl)-5,6-dihydro-2 H-[1,2]thiazine 1,1-dioxide (5g). Yellow solid, yield 75\%; $\operatorname{mp~} 136^{\circ} \mathrm{C}-137^{\circ} \mathrm{C}$; IR (KBr) $1605,1512,1460 \mathrm{~cm}^{-1} ;{ }^{1} \mathrm{H}$ NMR (300 MHz, $\mathrm{CDCl}_{3}$ ): $\delta 2.98\left(\mathrm{~s}, 3 \mathrm{H} .-\mathrm{SO}_{2} \mathrm{CH}_{3}\right), 3.83$ $\left(\mathrm{s}, 3 \mathrm{H},-\mathrm{OCH}_{3}\right), 4.55\left(\mathrm{~d}, J=10.5 \mathrm{~Hz}, 1 \mathrm{H}, \mathrm{H}^{1}\right), 4.76(\mathrm{dd}, J$ $\left.=10.5 \& 3.2 \mathrm{~Hz}, 1 \mathrm{H}, \mathrm{H}^{2}\right), 5.82\left(\mathrm{~d}, J=3.2 \mathrm{~Hz}, 1 \mathrm{H}, \mathrm{H}^{3}\right)$, $6.38\left(\mathrm{~d}, J=16.0 \mathrm{~Hz}, 1 \mathrm{H}, \mathrm{H}^{4}\right), 6.59(\mathrm{~d}, J=16.0 \mathrm{~Hz}, 1 \mathrm{H}$, $\left.\mathrm{H}^{5}\right), 6.83(\mathrm{~d}, J=8.7 \mathrm{~Hz}, 2 \mathrm{H}, \operatorname{ArH}), 6.97$ (d, $J=8.7 \mathrm{~Hz}$, $2 \mathrm{H}, \mathrm{ArH}), 6.99$ - $7.45(\mathrm{~m}, 8 \mathrm{H}, \mathrm{ArH}) \mathrm{ppm} ;{ }^{13} \mathrm{C} \mathrm{NMR}$ $\left(\mathrm{CDCl}_{3}\right): \delta 42.6(-\mathrm{CH}), 43.9\left(-\mathrm{SO}_{2} \mathrm{CH}_{3}\right), 55.4\left(-\mathrm{OCH}_{3}\right)$, 76.2 (-CH), 96.0, 114.1, 114.4, 120.3, 122.6, 125.3, $126.8,127.8,128.8,129.5,131.2,131.9,133.4,135.2$, 154.2, 159.6 ppm; MS (EI) $m / z: 563\left(\mathrm{M}^{+}\right)$. Anal. Calcd. for $\mathrm{C}_{26} \mathrm{H}_{23} \mathrm{Cl}_{2} \mathrm{NO}_{5} \mathrm{~S}_{2}$ : C, 55.32; H, 4.11; N, 2.48\%. Found: C, 55.26; H, 4.22; N, 2.52\%.

5-(4-Chloro-phenyl)-3-[2-(4-chloro-phenyl)-vinyl]-6 -methanesulfonyl-2-p-tolyl-5,6-dihydro-2H-[1,2]thiazine 1,1-dioxide (5h). Pale yellow solid, yield $72 \%$; mp $132^{\circ} \mathrm{C}$ - $133^{\circ} \mathrm{C}$; IR (KBr) 1603, 1510, $1460 \mathrm{~cm}^{-1}$; ${ }^{1} \mathrm{H}$ NMR $\left(300 \mathrm{MHz}, \mathrm{CDCl}_{3}\right): \delta 2.21\left(\mathrm{~s}, 3 \mathrm{H},-\mathrm{CH}_{3}\right), 3.01(\mathrm{~s}, 3 \mathrm{H}$. $\left.-\mathrm{SO}_{2} \mathrm{CH}_{3}\right), 4.53\left(\mathrm{~d}, J=10.5 \mathrm{~Hz}, 1 \mathrm{H}, \mathrm{H}^{1}\right), 4.71(\mathrm{dd}, J=$ $\left.10.5 \& 3.1 \mathrm{~Hz}, 1 \mathrm{H}, \mathrm{H}^{2}\right), 5.80\left(\mathrm{~d}, J=3.1 \mathrm{~Hz}, 1 \mathrm{H}, \mathrm{H}^{3}\right)$, $6.36\left(\mathrm{~d}, J=16.0 \mathrm{~Hz}, 1 \mathrm{H}, \mathrm{H}^{4}\right), 6.53(\mathrm{~d}, J=16.0 \mathrm{~Hz}, 1 \mathrm{H}$, $\left.\mathrm{H}^{5}\right), 6.90(\mathrm{~d}, J=8.7 \mathrm{~Hz}, 2 \mathrm{H}, \mathrm{ArH}), 7.06(\mathrm{~d}, J=8.7 \mathrm{~Hz}$, $2 \mathrm{H}, \mathrm{ArH}), 7.13$ - 7.49 (m, 8H, ArH) ppm; ${ }^{13} \mathrm{C} \mathrm{NMR}$ $\left(\mathrm{CDCl}_{3}\right): \delta 21.0\left(-\mathrm{CH}_{3}\right), 42.6(-\mathrm{CH}), 44.4\left(-\mathrm{SO}_{2} \mathrm{CH}_{3}\right)$, $76.6(-\mathrm{CH}), 96.0,114.0,114.3,120.5,123.4,123.8$, $125.4,127.1,127.8,131.7,131.9,133.4,133.9,134.5$, 142.5, 159.8 ppm; MS (EI) $m / z: 547\left(\mathrm{M}^{+}\right)$. Anal. Calcd. for $\mathrm{C}_{26} \mathrm{H}_{23} \mathrm{Cl}_{2} \mathrm{NO}_{4} \mathrm{~S}_{2}$ : C, 56.93; $\mathrm{H}, 4.23 ; \mathrm{N}, 2.55 \%$. Found: C, 57.03; H, 4.29; N, 2.61\%.

5-(4-Chloro-phenyl)-3-[2-(4-chloro-phenyl)-vinyl]-6 -methanesulfonyl-2-phenyl-5,6-dihydro-2H-[1,2]thiazine 1,1-dioxide (5i): Colorless solid, yield $78 \%$; mp $116^{\circ} \mathrm{C}$ $117^{\circ} \mathrm{C}$; IR (KBr) 1604, 1510, $1460 \mathrm{~cm}^{-1} ;{ }^{1} \mathrm{H}$ NMR $(300$ $\mathrm{MHz}, \mathrm{CDCl}_{3}$ ): $\delta 2.99$ (s, 3H. $-\mathrm{SO}_{2} \mathrm{CH}_{3}$ ), 4.51 (d, $J=10.4$ $\left.\mathrm{Hz}, 1 \mathrm{H}, \mathrm{H}^{1}\right), 4.71\left(\mathrm{dd}, J=10.4 \& 3.1 \mathrm{~Hz}, 1 \mathrm{H}, \mathrm{H}^{2}\right), 5.82$ $\left(\mathrm{d}, J=3.1 \mathrm{~Hz}, 1 \mathrm{H}, \mathrm{H}^{3}\right), 6.33\left(\mathrm{~d}, J=16.0 \mathrm{~Hz}, 1 \mathrm{H}, \mathrm{H}^{4}\right)$, $6.50\left(\mathrm{~d}, J=16.0 \mathrm{~Hz}, 1 \mathrm{H}, \mathrm{H}^{5}\right), 6.69-7.49(\mathrm{~m}, 15 \mathrm{H}, \mathrm{ArH})$ ppm; ${ }^{13} \mathrm{C} \mathrm{NMR}\left(\mathrm{CDCl}_{3}\right): \delta 43.0(-\mathrm{CH}), 44.9\left(-\mathrm{SO}_{2} \mathrm{CH}_{3}\right)$, $76.8(-\mathrm{CH}), 96.0,114.1,114.3,120.9,122.2,122.9$, $123.8,126.6,129.2,131.4,132.3,133.2,133.6,134.4$, 143.1, 159.7 ppm; MS (EI) m/z: $533\left(\mathrm{M}^{+}\right)$. Anal. Calcd. for $\mathrm{C}_{25} \mathrm{H}_{21} \mathrm{Cl}_{2} \mathrm{NO}_{4} \mathrm{~S}_{2}$ : C, 56.18; H, 3.96; N, 2.62\%. Found: C, 56.26; H, 4.09; N, 2.53\%.

\section{Acknowledgements}

Dr. P. Singh gratefully acknowledges the Durban University of Technology (DUT) and National Research Foundation (NRF) for the financial assistance. Spectral assistance from the Department of Chemistry, GNDU, Amritsar, is gratefully acknowledged.

\section{REFERENCES}

[1] B. Groenendaal, E. Ruijter and R. V. A. Orru, "1-Azadienes in Cycloaddition and Multicomponent Reactions towards N-heterocycles," Journal of Chemical Society, Chemical Communications, No. 43, 2008, pp. 5474-5489. doi:10.1039/b809206k 
[2] M. Behforouz and M. Ahmadian, "Diels-Alder Reactions of 1-Azadienes," Tetrahedron, Vol. 56, No. 30, 2000, pp. 5259-5288. doi:10.1016/S0040-4020(00)00259-3

[3] S. Jayakumar, M. P. S. Ishar and M. P. Mahajan, "Recent Advances in Synthetic Applications of Azadienes" Tetrahedron, Vol. 58, No. 3, 2002, pp. 379-471. doi:10.1016/S0040-4020(01)01050-X

[4] T. N. Danks and D. Velo-Rego, "Reaction of a Chromium Carbene Complex with 1-Azadienes and Synthesis of Trisubstituted Pyrroles," Tetrahedron Letters, Vol. 35, No. 50, 1994, pp. 9443-9444. doi:10.1016/S0040-4039(00)78565-0

[5] J. Barluenga, M. Tomas, J. A. Lopez-Pelegrin and E. Rubio, "Stereselective Cyclopropanation of 1-Azadienes with Fischer Carbine Complexes," Journal of Chemical Society, Chemical Communications, No. 6, 1995, pp. 665666. doi:10.1039/c39950000665

[6] Y. N. Romaschin, M. T. H. Liu and R. J. Bonneau, "The Facile Synthesis of 1,2,3-Trisubstituted Pyrroles from the Reaction of Chlorocarbenes with 1-Azabuta-1,3-dienes," Journal of Chemical Society, Chemical Communications, No. 5, 1999, pp. 447-448. doi:10.1039/a809508f

[7] M. Chagr, H. Fillion and A. Pougny, "A Regioselective Synthesis of 4,5- and 4,8-Disubstitutedazaanthraquinones by the Diels-Alder Route," Tetrahedron Letters, Vol. 29, No. 46, 1988, pp. 5913-5916. doi:10.1016/S0040-4039(00)82224-8

[8] D. Berger and W. Imhof, "One-Pot Synthesis of $\gamma$-Lactams in a Reaction Cascade from $\alpha, \beta$-Unsaturated Imines, $\mathrm{CO}$ and Ethylene Catalysed by $\mathrm{Ru}_{3}(\mathrm{CO})_{12}$," Journal of Chemical Society, Chemical Communications, No. 16, 1999, pp. 1457-1458. doi:10.1039/a903266e

[9] J. Barluenga, I. Merino and S. Palacios, "One Pot Synthesis of 2-Vinyl-1-azadienes and Divinylketones," Tetrahedron Letters, Vol. 30, No. 40, 1989, pp. 5493-5496. doi:10.1016/S0040-4039(01)80602-X

[10] N. Martin, A. M. Grau, L. Sanchez, C. Seoane and M. Torres, "The First Hetero-Diels-Alder Reaction of $60^{\circ} \mathrm{C}$ with 1-Azadienes. Synthesis of Tetrahydropyrido [2',3': 1,2][60]fullerene Derivatives," Journal of Organic Chemistry, Vol. 63, No. 22, 1998, pp. 8074-8076.

[11] C. R. Berry and R. P. Hsung, "Inverse Electron-Demand Aza-[4 + 2] Cycloaddition Reactions of Allenamides," Tetrahedron, Vol. 60, No. 35, 2004, pp. 7629-7636. doi:10.1016/j.tet.2004.05.117

[12] R. C. Clark, S. Pfeiffer and D. L. Boger, "Diastereoselective Diels-Alder Reactions of N-Sulfonyl-1-aza-1,3-butadienes with Optically Active Enol Ethers: An Asymmetric Variant of the 1-Azadiene Diels-Alder Reaction," Journal of American Chemical Society, Vol. 128, No, 8, 2006, pp. 2587-2593. doi:10.1021/ja0571646

[13] M. He, R. Struble and J. W. Bode, "Highly Enantioselective Azadiene Diels-Alder Reactions Catalyzed by Chiral N-Heterocyclic Carbenes," Journal of American Chemical Society, Vol. 128, No. 26, 2006, pp. 8418-8420. doi:10.1021/ja062707c

[14] A. Robin, K. Julienne, J. C. Meslin and D. Deniaud, "Synthesis of Pyridone and Pyridine Rings by [4 + 2] Hetero-Cyclocondensation," Tetrahedron Letters, Vol. 45, No. 52, 2004, pp. 9557-9559. doi:10.1016/j.tetlet.2004.10.142

[15] M. C. Elliott, E. Kruiswijk and D. J. Willock, “Asymmetric Hetero-Diels-Alder Reactions. Reactions of Oxazolo [3,2-c]pyrimidines," Tetrahedron, Vol. 57, No. 51, 2001, pp. 10139-10146. doi:10.1016/S0040-4020(01)01032-8

[16] D. L. Boger, "Diels-Alder Reactions of Azdienes," Tetrahedron, Vol. 39, No. 18, 1983, pp. 2869-2939. doi:10.1016/S0040-4020(01)92154-4

[17] D. L. Boger and S. M. Weinreb, "In Hetero Diels-Alder Methodology in Organic Synthesis," Academic Press Inc., San Diego, 1987, p 239.

[18] S. N. Mazumdar, M. Sharma and M. P. Mahajan, "DielsAlder Cycloaddition Reactions of 1,3-Diazabutadienes with Sulfene," Tetrahedron Letters, Vol. 28, No. 23, 1987, pp. 2641-2642. doi:10.1016/S0040-4039(00)96169-0

[19] P. Singh, G. Bhargava and M. P. Mahajan, "Tandem [2+ 2] Cycloaddition and Cope Rearrangement in Reactions of Cross-Conjugated Azatrienes with Conjugated Ketenes: A Facile Single Step Synthesis of Novel Azocinone Derivatives," Tetrahedron, Vol. 62, No. 48, 2006, pp. 11267 11273. doi:10.1016/i.tet.2006.09.007

[20] M. H. Elnagdi, S. M. Mousawi, M. M. Abdel-Khalik, S. El-Sheriny and E. John, "Studies with Enaminones: Reactivity of 1,5-Disubstituted-1,4-pentadien-3-ones toward Electrophilic Reagents. A Novel Route to Azolylazines, Benzofuranals, Pyranones," Journal of Heterocyclic Chemistry, Vol. 38, No. 4, 2001, pp. 949-953. doi: $10.1002 /$ jhet. 5570380422 